\title{
Rotational properties of the Orion nebular cluster revised
}

\author{
Natália R. Landin ${ }^{1}$, Paolo Ventura ${ }^{2}$, Francesca D'Antona ${ }^{2}$, \\ Luiz T. S. Mendes ${ }^{1,3}$ and Luiz P. R. Vaz ${ }^{1}$ \\ ${ }^{1}$ Depto de Física - ICEx - UFMG, Av. Antonio Carlos, 6627 - Brazil \\ email: nlandin,luizt,lpv@fisica.ufmg.br \\ ${ }^{2}$ Osservatorio Astronomico di Roma, Via Frascati, 33 - Italy \\ ${ }^{3}$ Depto de Engenharia Eletrônica - CPDEE - UFMG, Av. Antonio Carlos, 6627 - Brazil
}

\begin{abstract}
The observational data of the Orion Nebula Cluster (ONC) is reanalyzed by means of new sets of pre-main sequence (PMS) evolutionary tracks including rotation, non-gray boundary conditions (BC's) and either low (LCE) or high convection efficiency (HCE), aiming better understanding of the appropriate physical constraints for the rotational evolution of the stars within the ONC. The role played by convection is a key aspect of our analysis, since there are conflicting results from theory and observations. We derived stellar masses and ages for the ONC by using both LCE and HCE and considered was the role of non-gray atmospheres. Our results show that the resulting mass distribution for the bulk of the ONC population is in the range $0.2-0.4 \mathrm{M}_{\odot}$ for our non-gray models, and in the range $0.1-0.3 \mathrm{M}_{\odot}$ for gray models. In agreement with previous works, we found that a large percentage $(\sim 70 \%)$ of low-mass stars $\left(\mathrm{M} \leqslant \mathrm{M}_{\mathrm{tr}}\right.$, where $\mathrm{M}_{\mathrm{tr}}$ is a transition mass) in the ONC appears to be fast rotators ( $\mathrm{P}<4$ days $) . \mathrm{M}_{\mathrm{tr}}$ depends on the model choosen, being $\mathrm{M}_{\mathrm{tr}}=0.5$ for $\mathrm{LCE}, \mathrm{M}_{\mathrm{tr}}=0.35$ for $\mathrm{HCE}$ and, as found in previous works, $\mathrm{M}_{\mathrm{tr}}=0.25$ for gray models. Finally, our analysis indicates that a second parameter is needed for a proper description of convection in the PMS phase.
\end{abstract}

Keywords. Stars: evolution, interiors, rotation, Hertzsprung Russell and C-M diagrams

\section{Introduction}

We generated new sets of PMS models with the ATON2.4 stellar evolution code (Landin et al. 2006), which includes rotation and non-gray BC's. Using observational data, kindly provided by Stassun (Stassun et al. 1999, 2004), and our theoretical predictions, we will try to get a better understanding of the physical constraints to be used for a general description of stellar structure and evolution stars within the ONC by means of our new sets of LCE and HCE models.

We computed two HCE models (the $\alpha=2.0$ set allows a fit to the solar radius and that with $\alpha=2.2$ should provide very efficient convection set) and one LCE set with $\alpha=1.0$, that leads to a better agreement with the $\mathrm{Li}$ vs. $\mathrm{T}_{\text {eff }}$ relation observed in young open clusters stars (Landin et al. 2006). We assigned to each observed point masses and ages. The masses derived with HCE sets are systematically smaller than with LCE models, hence yielding younger ages.

\section{Comparison with the ONC stars}

The resulting distribution for the bulk of the ONC population is in the range of $0.2-$ $0.4 \mathrm{M}_{\odot}$ for our new non-gray models, while being $0.1-0.3 \mathrm{M}_{\odot}$ for models having gray BC's. The mass function for the $\alpha 1.0$ set peaks in the mass interval $0.3-0.4 \mathrm{M}_{\odot}$, but we note the presence of two significant groups of stars with masses in the ranges $0.2-0.3 \mathrm{M}_{\odot}$ and $0.6-0.9 \mathrm{M}_{\odot}$. For the HCE models this latter group of objects becomes less relevant and 
the mass function peaks in the range $0.2-0.4 \mathrm{M}_{\odot}$. A discussion on the mass assignments can be found in Landin et al. (2006). The age distribution depends on the choice of $\alpha$. A slightly younger population is obtained as $\alpha$ increases, and, for HCE cases, a very young group of stars appears at ages $\sim 1-2 \times 10^{5} \mathrm{yr}$, but not being present for the LCE models.
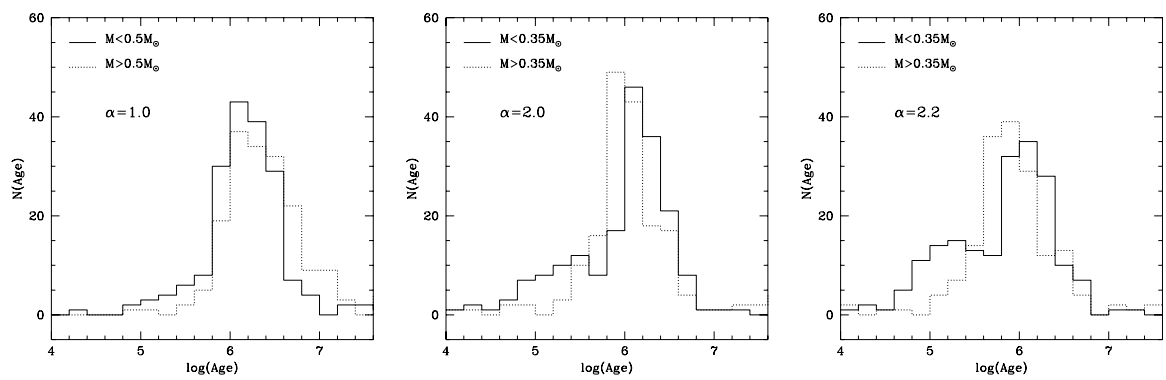

Figure 1. The age distributions of the ONC stars for $M<M_{t r}$ and $M>M_{t r}$.

We define a transition mass, $\mathrm{M}_{\mathrm{tr}}$, on the basis of the rotational period distribution. In Fig. 1, we compare the age distribution for two different mass ranges, $M>M_{t r}$ and $\mathrm{M}<\mathrm{M}_{\mathrm{tr}}$. For the $\alpha 1.0$ set, the two populations have a similar distribution. On the contrary, the age distribution of the two HCE groups is very different. Thus, while the existence of a group of younger stars would be possible in the formation history of the ONC, it should be present for any mass. The discrepancy in the age distribution may be another indication that the $\alpha 1.0$ set provides a better description of PMS stars, in agreement with the Li depletion, nevertheless 2-D hydrodynamic simulations predict HCE for PMS. In any case, this certainly is not final and we proceed with the analysis by using the three sets of tracks. However, we keep in mind that the comparisons made indicate that convection in PMS may be affected by a second parameter.

The overall period distribution of ONC stars has a bimodal character, showing a primary peak corresponding to fast rotators and a secondary peak of slow rotators, see Fig. 5 of Landin et al. (2006). The former can be associated with spin up due to conservation of the total angular momentum. The latter indicates the presence of a mechanism preventing stellar spinning up, at least in the early evolutionary phases.
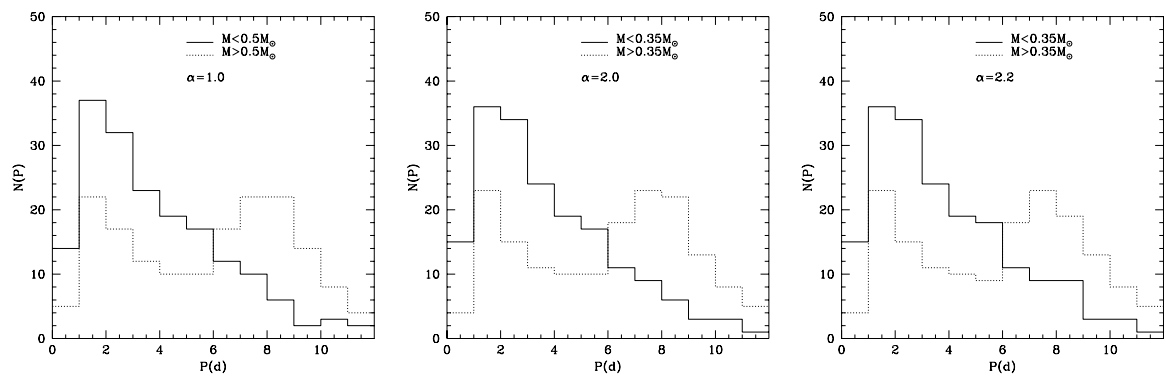

Figure 2. The dependence on mass of the period distribution of the ONC objects.

The rotational properties of the ONC stars vary with mass: stars with $M>M_{t r}$ have a clearly bimodal distribution, while those with $\mathrm{M}<\mathrm{M}_{\mathrm{tr}}$ contain only a tail of slow rotators. Although the dichotomy does not depend neither on $\alpha$ nor on BC's, $\mathrm{M}_{\mathrm{tr}}$ varies according to them, being $0.5,0.35$ and $0.25 \mathrm{M}_{\odot}$ for LCE, HCE and gray models, respectively.

In Fig. 2 we show the histogram of periods for stars less and more massive than $\mathrm{M}_{\mathrm{tr}}$. The secondary peak at $\mathrm{P} \sim 8 \mathrm{~d}$, already seen in the overal period distribution, is present 
only in the population at $\mathrm{M}>\mathrm{M}_{\mathrm{tr}}$, while the low mass objects show a clear trend towards short periods. We found that $\sim 70 \%$ of low-mass stars $\left(\mathrm{M}<\mathrm{M}_{\mathrm{tr}}\right)$ appears to be fast rotators $\left(\mathrm{P}<4\right.$ days) and only $20 \%$ of masses $<\mathrm{M}_{\mathrm{tr}}$ and $\sim 54 \%$ of the masses $>\mathrm{M}_{\mathrm{tr}}$ have $\mathrm{P}>6$ days. This dichotomy indicates that either (i) disk locking is responsible for the presence of the secondary peak, and stars with $\mathrm{M}>\mathrm{M}_{\mathrm{tr}}$ tend to be embedded in their disk longer than their low mass counterparts, (ii) the locking time is similar, but the stars with $\mathrm{M}>\mathrm{M}_{\mathrm{tr}}$ evolve faster and a larger fraction of their PMS lifetime is locked, or (iii) the locking period of the group with $\mathrm{M}<\mathrm{M}_{\mathrm{tr}}$ is significantly lower than $\sim 8 \mathrm{~d}$. Following the suggestion by Herbst et al. (2002), that the longer period peak indicates that some stars are locked on their disks with $\mathrm{P} \geqslant 8$ days $\left(\mathrm{P}_{\text {tsh }}\right)$, we established the following criterion: stars with $\mathrm{P} \geqslant \mathrm{P}_{\text {tsh }}$ are locked, stars with $\mathrm{P}<\mathrm{P}_{\text {tsh }}$ are unlocked. For the latter group we determined the epoch at which their period was equal to 8 days. This would be the time at which the stars would have lost their disks. In this way, we identify three distinct populations: (1) early fast rotators (stars locked only for ages $<10^{5} \mathrm{yr}$ ), (2) slow rotators (stars probably still disk embedded) and (3) moderate rotators.
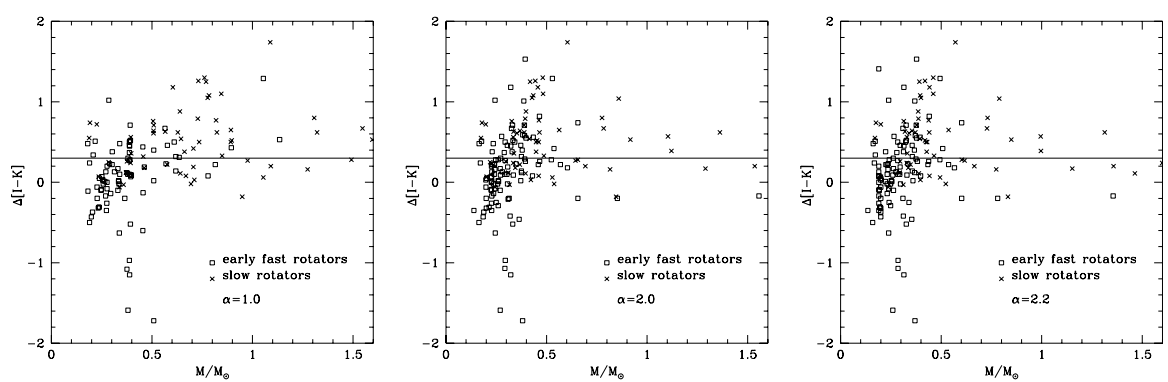

Figure 3. The observed IR excess of our sample stars plotted against their inferred mass.

In our analysis we use the rotation period as an indicator of the presence of a disk. In order to test the reliability of this hypothesis, we should use, at least, one observational indicator of presence of disk and/or accretion, like the infrared (IR) excess $\Delta[\mathrm{I}-\mathrm{K}]$. It is expected that still locked stars have $\Delta[\mathrm{I}-\mathrm{K}]>0.3$, and those that evolved without disk should have IR excess significantly lower than this threshold value. We report in Fig. 3 the observed stars on the plane $\Delta[\mathrm{I}-\mathrm{K}]$ vs. mass. We can see that sources that we identified as still locked (slow rotators $-\times$ ) are mainly concentrated above the $\Delta[I-K]=0.3$ line, while those that evolved without a disk (early fast rotators) lie mainly below it. The evolutionary tracks and isochrones are available from www.mporzio.astro.it/ tsa

\section{Acknowledgements}

The authors thank Dr. K. Stassun for providing the ONC data. NRL thank the Osservatorio Astronomico di Roma for their hospitality during an extended visit. Financial support from the Brazilian agencies CAPES, CNPq, FAPEMIG and FUNDEP is acknowledged.

\section{References}

Herbst, W., Bailer-Jones, C.A.L., Mundt, R., Meisenheimer, K., \& Wackermann, R., 2002, A\&A, 396,513

Landin, N.R., Ventura, P., D'Antona, F., Mendes, L.T.S., \& Vaz, L.P.R., 2006, A\&A 456, 269

Stassun, K.G., Mathieu, R.D., Mazeh, T., \& Vrba, F.J., 1999, AJ, 117, 2941

Stassun, K.G., Ardila, D.R., Basri, G., \& Mathieu R.D., 2004, AJ, 127, 3537 